\title{
Metastatic PRAME-Expressing Juvenile Spitzoid Melanoma on the Buttock
}

\author{
Yusuke Muto Taku Fujimura Yumi Kambayashi Kentaro Ohuchi \\ Ryo Amagai Akira Hashimoto Setsuya Aiba \\ Department of Dermatology, Tohoku University Graduate School of Medicine, Sendai, \\ Japan
}

\section{Keywords}

PRAME · Spitzoid melanoma · Pediatric melanoma - Immunohistochemical staining ·

Diagnostic methods

\begin{abstract}
Since the cost of molecular biological methods for Spitzoid neoplasms is expensive, the number of institutes that employ these methods might be limited. Preferentially expressed antigen in melanoma (PRAME) is a tumor-associated antigen that is useful to distinguish melanoma from other melanocytic disorders, including pediatric Spitzoid tumors that are difficult to diagnose by conventional methods alone. In this report, we report a case of PRAME-expressing juvenile Spitzoid melanoma with lymph node metastasis. Unexpectedly, there were few PRAME-expressing cells in the primary tumor, whereas most metastatic tumors expressed PRAME in the metastatic lymph node. These observations might suggest that, in Spitzoid melanomas, a limited number of melanoma cells possess metastatic potential and that metastatic lesions possess clonality.

\section{Introduction}

Preferentially expressed antigen in melanoma (PRAME) is a tumor-associated antigen that was first identified through analysis of the specificity of tumor-reactive T-cell clones derived from a patient with metastatic cutaneous melanoma [1]. Since the number of institutes that can perform these methods might be limited, PRAME is useful to distinguish 


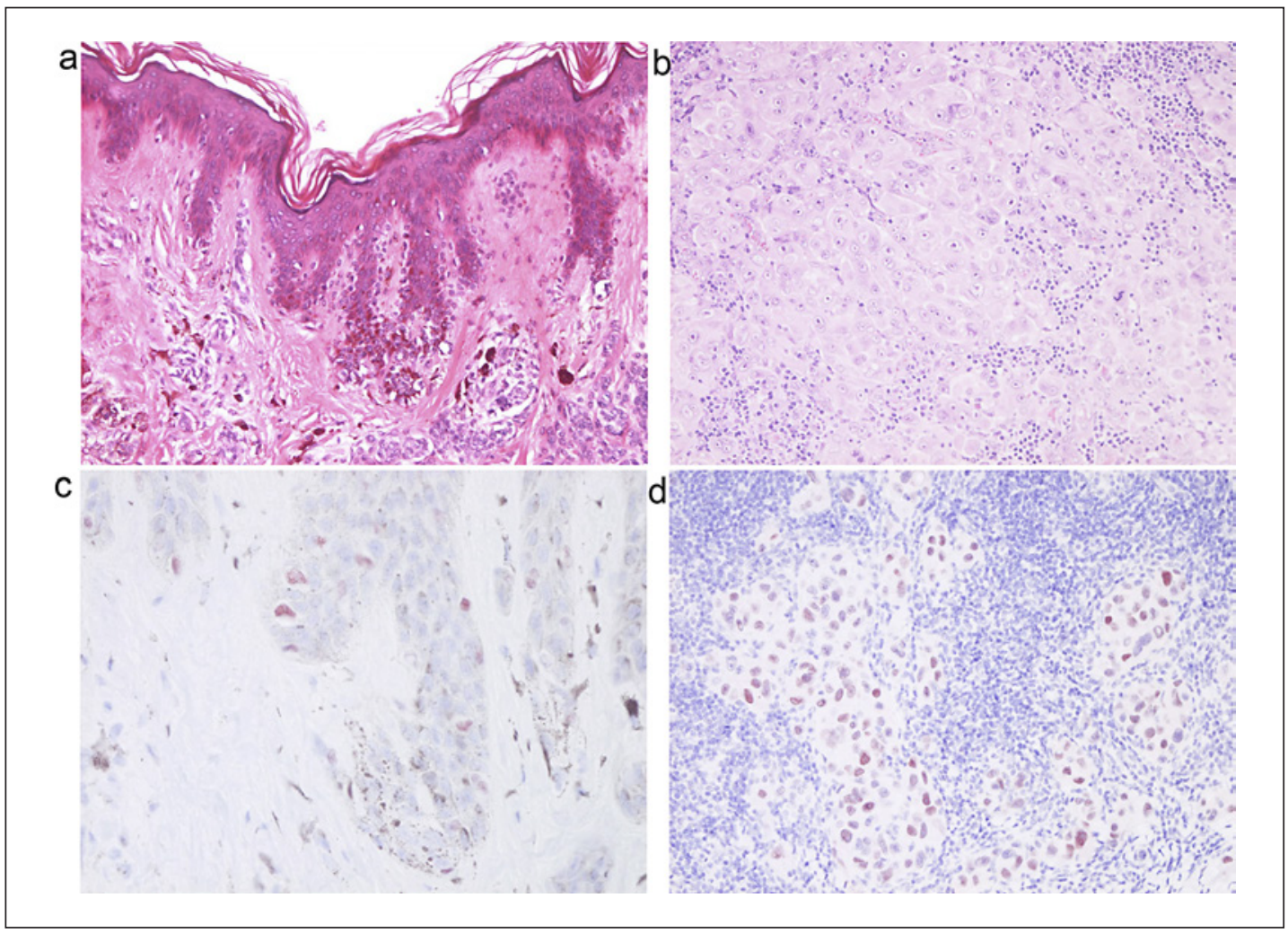

Fig. 1. Histological findings of the primary tumor (a) and metastatic lymph node (b): atypical epithelioid and spindle-shaped melanocytes with large hyperchromatic pleomorphic nuclei were observed. Immunohistochemical staining of PRAME for the primary tumor (c) and metastatic lymph node (d).

melanoma from other melanocytic disorders (e.g., Spitz nevus) that are difficult to diagnose by conventional methods alone [2]. In this report, we describe a case of PRAME-expressing Spitzoid melanoma on the buttock that metastasized to the inguinal lymph nodes.

\section{Case Report}

A 14-year-old Japanese girl visited our outpatient clinic with a history of resection of a tumor on the buttock as a pyogenic granuloma. Her initial diagnosis by a pathologist was Spitz nevus, but a plastic surgeon was consulted at our outpatient clinic to obtain a second opinion. Histological findings of the primary tumor revealed atypical epithelioid and spindle-shaped melanocytes with large hyperchromatic pleomorphic nuclei in the epidermis and superficial dermis (Fig. 1a). Immunohistochemical staining (IHC) revealed that these atypical epithelioid and spindle-shaped melanocytes were diffusely positive for Melan A and S100, but positive for HMB45 only at the basement membrane zone (data not shown). Then, we performed extended resection and sentinel lymph node biopsy at the inguinal lymph node to rule out the possible diagnosis of Spitzoid melanoma. Since we detected metastatic melanoma at the sentinel lymph node (Fig. 1b), we performed further dissection of the right inguinal lymph nodes, which revealed no additional metastasis. From the above findings, our diagnosis was Spitzoid melanoma with single lymph node metastasis. The patient was treated with 3 million units of interferon- $\beta$ every month as an adjuvant therapy for 5 years after the surgical 
treatment in order to induce melanoma-specific CD8+ T cells as well as HLA-DR+ dendritic cells in both locoregional lesions of the inguinal lymph nodes $[3,4]$. There was no evidence of metastasis 8 years after the radical dissection of the right inguinal lymph nodes.

To retrospectively analyze the diagnosis of Spitzoid melanoma, we employed IHC of PRAME for the primary tumor and metastatic lymph node, which suggested that, similar to the HMB45 expression, a few PRAME-expressing atypical epithelioid and spindle-shaped melanocytes were detected only at the basement membrane zone of the primary tumor (Fig. 1c), whereas most of the atypical epithelioid and spindle-shaped melanocytes expressed PRAME (Fig. 1d).

\section{Discussion}

Since the diagnosis of primary Spitzoid melanoma by conventional methods (for example, conventional ABCDE [asymmetry, border irregularity, color variation, diameter $>6 \mathrm{~mm}$, and evolution] criteria, dermoscopy, histopathological diagnosis by HE staining) is challenging [2, 5], recently, several diagnostic tools were developed [5-10]. Indeed, Bahrami et al. [5] reviewed the molecular biology of pediatric melanoma, including Spitzoid melanoma, suggesting that the specific kinase gene fusion (including NTRK1, NTRK3, ALK, ROS1, RET, MET, and BRAF) might be useful for the diagnosis of Spitzoid melanoma [5-7]. A chromosomal copy number status detected by fluorescence in situ hybridization or complete genomic hybridization could also be useful $[5,8]$.

Although these molecular biological methods are useful for the diagnosis of Spitzoid neoplasm, institutes that can employ these expensive methods are limited. Notably, Lezcano et al. [2] evaluated the IHC of PRAME for challenging melanocytic tumors, including Spitzoid melanoma, by cytogenetic test, and the result suggested the high sensitivity (75.0\%) and specificity (98.8\%) of PRAME IHC for the diagnosis of Spitzoid melanoma. This report suggested the use of PRAME IHC as a supportive test in the evaluation of pediatric melanoma including Spitzoid melanoma [2]. From the above findings, in this report, we employed IHC staining of PRAME for a case of juvenile Spitzoid melanoma with lymph node metastasis. Unexpectedly, there were few PRAME-expressing cells in the primary tumor, whereas most metastatic tumors expressed PRAME in the metastatic lymph node. These observations might suggest that limited numbers of melanoma cells possess metastatic potential, and that, in Spitzoid melanoma, metastatic lesions possess clonality. Since we present a single case only, further cases are needed to confirm the pathogenesis of metastatic PRAME-expressing Spitzoid melanoma.

\section{Statement of Ethics}

The description of the case is retrospective, and the person involved remained completely anonymous. The patient in this paper has given written informed consent to the publication of her case details (including images).

\section{Conflict of Interest Statement}

The authors have no conflicts of interests to declare. 


\section{Funding Sources}

There are no funding sources.

\section{Author Contributions}

T. Fujimura designed the research study. Y. Muto, T. Fujimura, Y. Kambayashi, K. Ohuchi, R. Amagai, and A. Hashimoto treated the patient and acquired the clinical data. Y. Muto and T. Fujimura wrote the manuscript. Y. Muto performed IHC. T. Fujimura and S. Aiba supervised the study.

\section{References}

1 Lezcano C, Jungbluth AA, Nehal KS, Hollmann TJ, Busam KJ. PRAME Expression in Melanocytic Tumors. Am J Surg Pathol. 2018;42(11):1456-65.

2 Lezcano C, Jungbluth AA, Busam KJ. Comparison of Immunohistochemistry for PRAME with Cytogenetic Test Results in the Evaluation of Challenging Melanocytic Tumors. Am J Surg Pathol. 2020;44(7):893-900.

3 Fujimura T, Okuyama R, Ohtani T, Ito Y, Haga T, Hashimoto A, et al. Perilesional treatment of metastatic melanoma with interferon-beta. Clin Exp Dermatol. 2009;34(7):793-9.

4 Kakizaki A, Fujimura T, Furudate S, Kambayashi Y, Yamauchi T, Yagita H, et al. Immunomodulatory effect of peritumorally administered interferon-beta on melanoma through tumor-associated macrophages. Oncoimmunology. 2015;4(11):e1047584.

5 Bahrami A, Barnhill RL. Pathology and genomics of pediatric melanoma: A critical reexamination and new insights. Pediatr Blood Cancer. 2018;65.

6 Lee S, Barnhill RL, Dummer R, Dalton J, Wu J, Pappo A, et al. TERT Promoter Mutations Are Predictive of Aggressive Clinical Behavior in Patients with Spitzoid Melanocytic Neoplasms. Sci Rep. 2015;5:11200.

7 Wiesner T, He J, Yelensky R, Esteve-Puig R, Botton T, Yeh I, et al. Kinase fusions are frequent in Spitz tumours and spitzoid melanomas. Nat Commun. 2014;5:3116.

8 Ali L, Helm T, Cheney R, Conroy J, Sait S, Guitart J, et al. Correlating array comparative genomic hybridization findings with histology and outcome in spitzoid melanocytic neoplasms. Int J Clin Exp Pathol. 2010;3(6): 593-9.

9 Lang UE, Yeh I, McCalmont TH. Molecular Melanoma Diagnosis Update: Gene Fusion, Genomic Hybridization, and Massively Parallel Short-Read Sequencing. Clin Lab Med. 2017;37(3):473-84.

10 Lallas A, Apalla Z, Ioannides D, Lazaridou E, Kyrgidis A, Broganelli P, et al.; International Dermoscopy Society. Update on dermoscopy of Spitz/Reed naevi and management guidelines by the International Dermoscopy Society. Br J Dermatol. 2017;177(3):645-55. 